\title{
HOMOCLINIC SOLUTIONS FOR LINEAR AND LINEARIZABLE ORDINARY DIFFERENTIAL EQUATIONS
}

\author{
CEZAR AVRAMESCU AND CRISTIAN VLADIMIRESCU
}

Received 2 July 2000

Using functional arguments, some existence results for the infinite boundary value problem $\dot{x}=F(t, x), x(-\infty)=x(+\infty)$ are given. A solution of this problem is frequently called, from Poincaré, homoclinic.

\section{Introduction}

Let $F: \mathbb{R} \times \mathbb{R}^{N} \rightarrow \mathbb{R}^{N}$ be a continuous function; consider the problem

$$
\begin{gathered}
\dot{x}=F(t, x), \\
x(-\infty)=x(+\infty),
\end{gathered}
$$

where

$$
x( \pm \infty):=\lim _{t \rightarrow \pm \infty} x(t) .
$$

Throughout this paper, the limits which appear are always finite; other cases are mentioned.

In a certain sense, the problem (1.1) and (1.2) may be considered as a generalization of the classical bilocal boundary value problem

$$
\dot{x}=F(t, x), \quad x(a)=x(b) .
$$

The problem (1.4), which is closely related to the problem of periodic solutions, has constituted the object of numerous fundamental studies; last year, a systematic study based on the theory of topological degree has been drawn up by J. Mawhin [17, 18, 19, 20]. In the cited works the reader can find a rich bibliography in this field.

On the other hand, the existence of solutions of (1.1) such that there exist $x( \pm \infty)$ (these solutions are often called convergent), has constituted the object of many works (see [3]). A substantial contribution in this field is due to A. G. Kartsatos [13, 14, 15, 16]; some of the ideas contained in his works will be used in this paper.

Copyright $\odot 2000$ Hindawi Publishing Corporation

Abstract and Applied Analysis 5:2 (2000) 65-83

2000 Mathematics Subject Classification: 34B05, 34B27

URL: http://aaa.hindawi.com/volume-5/S1085337500000300.html 
Most of the papers deal with the existence of the limit $x(+\infty)$; less are dedicated to the existence of both limits $x( \pm \infty)$; among these, we mention $[2,12]$.

Among the first results that consider the boundary value problems on noncompact intervals one counts the results obtained by A. G. Kartsatos [16].

In another direction, it was pointed out interest in the asymptotic behavior of solutions for integral equations, too; for the Volterra equations, the fundamental paper of C. Corduneanu [10] and for the Hammerstein equation the one of D. Petrovanu [21]. The existence problem of convergent solutions for the integral equations has been considered in $[4,5,6]$.

The boundary value problems of the type (1.1) and (1.2) has been considered in $[1,2,7,8]$.

In the present paper, the boundary value problem (1.1) and (1.2) is discussed in the case when the differential equation is linear or linearizable; through this last term we understand the fact that (1.1) may be reduced to another which contains a linear part.

Section 2 deals with the notations and the main hypotheses. Next we present some general results which will be used in what follows.

In Section 3, we prove some existence results for the problem (1.1) and (1.2) in the case when $F(t, x)=A(t) x+b(t)$; these results are determined by the specific properties of the matrix $A(t)$.

Finally, in Section 4, we give two existence theorems in the case when $F$ is linearizable.

\section{Notations and general results}

2.1. We denote by $\mathbb{R}^{N}$ the $N$-dimensional Euclidean space and by $\mu_{N}(\mathbb{R})$ the space of all $N \times N$ real matrices; both spaces are endowed with the norm $|\cdot|$ and the usual scalar product in $\mathbb{R}^{N}$ is $\langle\cdot, \cdot\rangle$.

The fundamental functional space is

$$
C_{c}=C_{c}\left(\mathbb{R}, \mathbb{R}^{N}\right):=\left\{x: \mathbb{R} \longrightarrow \mathbb{R}^{N}, x \text { continuous }\right\}
$$

endowed with the topology of uniform convergence on every compact interval of $\mathbb{R}$.

We consider the following linear subspaces of $C_{c}$ :

$$
\begin{aligned}
C & :=\left\{x \in C_{c}, x \text { bounded on } \mathbb{R}\right\}, \\
C_{l} & :=\left\{x \in C_{c}, \exists x( \pm \infty):=\lim _{t \rightarrow \pm \infty} x(t) \in \mathbb{R}^{N}\right\}, \\
C_{l l} & :=\left\{x \in C_{l}, x(+\infty)=x(-\infty)\right\}, \\
C_{0} & :=\left\{x \in C_{l}, x(+\infty)=x(-\infty)=0\right\} .
\end{aligned}
$$

All these spaces are Banach spaces with the norm

$$
\|x\|_{\infty}:=\sup _{t \in \mathbb{R}}|x(t)|
$$

their topology being stronger than the one induced by the topology of $C_{c}$. 
For every function $h$ defined on $\mathbb{R}$, we put

$$
h( \pm \infty):=\lim _{t \rightarrow \pm \infty} h(t)
$$

(the limits being considered finite).

Moreover, let

$$
\begin{aligned}
& C_{l}^{+}:=\left\{x \in C_{c}, \exists x(+\infty):=\lim _{t \rightarrow+\infty} x(t)\right\}, \\
& C_{l}^{-}:=\left\{x \in C_{c}, \exists x(-\infty):=\lim _{t \rightarrow-\infty} x(t)\right\} .
\end{aligned}
$$

An analogous signification have the notations $C_{l l}^{+}, C_{l l}^{-}, C_{0}^{+}, C_{0}^{-}$.

Another space is $L^{1}:=L^{1}\left(\mathbb{R}, \mathbb{R}^{N}\right)$, the space of all Lebesgue integrable functions on $\mathbb{R}$. Also, consider

$$
\begin{aligned}
& C_{(a, b)}:=\left\{x:[a, b] \longrightarrow \mathbb{R}^{N}, x \text { continuous }\right\}, \\
& C_{[a, b]}:=\left\{x \in C_{(a, b)}, x(a)=x(b)\right\} .
\end{aligned}
$$

These three Banach spaces are endowed with their usual norms. Finally, we put

$$
\mathbb{R}_{+}:=[0,+\infty), \quad \mathbb{R}_{-}:=(-\infty, 0], \quad \Sigma_{r}:=\left\{x \in \mathbb{R}^{N},|x| \leq r\right\} .
$$

2.2. The aim of this subsection is to point out some important properties of $C_{l}$.

Proposition 2.1. There exists an isometric isomorphism between the spaces $C_{l}$ and $C_{(a, b)}$.

Proof. Let $\varphi:(a, b) \rightarrow \mathbb{R}$ be a continuous strictly nondecreasing function, with $\lim _{t \searrow a} \varphi(t)$ $=-\infty, \lim _{t \nearrow b} \varphi(t)=+\infty$. The function $\Phi: C_{l} \rightarrow C_{(a, b)}$, defined by

$$
(\Phi x)(t):= \begin{cases}x(\varphi(t)), & t \in(a, b), \\ x(-\infty), & t=a, \\ x(+\infty), & t=b,\end{cases}
$$

is an isometric isomorphism between $C_{l}$ and $C_{(a, b)}$.

Remark 2.2. The same function $\Phi$ represents an isometric isomorphism between $C_{l l}$ and $\mathrm{C}_{[a, b]}$.

Definition 2.3 (see [3]). We say that a family $A \subset C_{l}$ is equiconvergent if

$$
\begin{gathered}
\forall \varepsilon>0, \quad \exists T=T(\varepsilon)>0, \\
\forall t^{\prime}, t^{\prime \prime} \in \mathbb{R}, \quad \text { with } t^{\prime} \cdot t^{\prime \prime}>0, \quad\left|t^{\prime}\right|>T, \quad\left|t^{\prime \prime}\right|>T, \\
\left|x\left(t^{\prime}\right)-x\left(t^{\prime \prime}\right)\right|<\varepsilon, \quad \forall x \in \mathscr{A} .
\end{gathered}
$$


Proposition 2.4. A family $\mathscr{A} \subset C_{l}$ is relatively compact if and only if the following conditions are satisfied:

(i) $A$ is uniformly bounded in $C$;

(ii) $A$ is equicontinuous in $C_{c}$;

(iii) $\mathscr{A}$ is equiconvergent.

Proof. From (ii) and (iii) one gets $\mathscr{A}$ is equicontinuous in $C_{l}$. Therefore, $\Phi(\mathscr{A})$ is an equicontinuous and uniformly bounded set in $C_{(a, b)}$. So, based on Ascoli-Arzéla theorem, we conclude that $\Phi(\mathscr{A})$ is relatively compact in $C_{(a, b)}$.

2.3. In what follows we will point out two useful properties. We state these properties on $\mathbb{R}_{+}$and $\mathbb{R}_{-}$.

Let $X$ be a Banach space and $P: X \rightarrow X$ be a projector.

Proposition 2.5. Let $U: \mathbb{R}_{+} \rightarrow X^{X}$ be a function which fulfills the following hypotheses:

(i) the function $t \mapsto U(t)$ is continuous on $\mathbb{R}_{+}$;

(ii) for every $t \in \mathbb{R}_{+}$there exists $U^{-1}(t): X \rightarrow X$ such that

$$
U^{-1}(t) U(t)=I_{X}
$$

(iii) the function $t \mapsto U^{-1}(t)$ is continuous on $\mathbb{R}_{+}$;

(iv) there exists $k>0$ such that

$$
\int_{0}^{+\infty}\left\|U(t) P U^{-1}(s) \xi\right\|_{X} d s \leq k, \quad \forall t \in \mathbb{R}_{+}, \xi \in \Sigma_{1} .
$$

Then, there exist two constants $\alpha, \beta>0$ such that

$$
\|U(t) P \xi\|_{X} \leq \alpha \cdot e^{-\beta t}, \quad \forall t \in \mathbb{R}_{+}, \xi \in \Sigma_{1} .
$$

Proof. We put

$$
z(t):=\int_{0}^{t} \frac{d s}{\|U(s) P \xi\|_{X}}
$$

From the identity

$$
z(t) U(t) P \xi=\int_{0}^{t} U(t) P U^{-1}(s) U(s) P \frac{1}{\|U(s) P \xi\|_{X}} d s,
$$

we get

$$
\|z(t) U(t) P \xi\|_{X}=z(t) \cdot\|U(t) P \xi\|_{X} \leq k, \quad \forall t \geq 0,
$$

hence

$$
\frac{\dot{z}(t)}{z(t)} \geq \frac{1}{k}
$$

Therefore,

$$
z(t) \geq z\left(t_{0}\right) \cdot e^{(1 / k)\left(t-t_{0}\right)}, \quad \forall t \geq t_{0}>0 .
$$


But, from (2.15) and (2.17) we deduce

$$
\|U(t) P \xi\|_{X} \leq \frac{k}{z(t)}, \quad \forall t \geq t_{0}>0 .
$$

So, Proposition 2.5 is proved.

Proposition 2.6. Suppose that the hypothesis (i) from Proposition 2.5 is true. Let $\xi \in X$ be such that $P \xi \neq 0$. If

$$
\int_{t}^{+\infty}\|U(s) P \xi\|_{X} \leq k, \quad \forall t>0,
$$

then

$$
\limsup _{t \rightarrow+\infty}\|U(t) P \xi\|_{X}=+\infty
$$

Proof. Indeed, if

$$
u(t):=\frac{1}{\|U(t) P \xi\|_{X}},
$$

then by using the relation

$$
\int_{(\cdot)}^{+\infty} u(t) d t<\infty
$$

it results that

$$
\liminf _{t \rightarrow+\infty} u(t)=0
$$

The conclusion is now obvious.

An important particular case is the one when

$$
U: \mathbb{R}_{+} \longrightarrow M_{N}(\mathbb{R})
$$

(see $[11,12])$.

Remark 2.7. A similar result may be obtained on the negative semiaxis $\mathbb{R}_{-}$.

\section{Linear equations}

3.1. Let $A: \mathbb{R} \rightarrow M_{N}(\mathbb{R})$ be a continuous function and $b \in C_{c}$; consider the equations

$$
\begin{gathered}
\dot{x}=A(t) x, \\
\dot{x}=A(t) x+b(t) .
\end{gathered}
$$

We denote

$$
\begin{gathered}
\mathscr{X}:=\left\{x \in C_{c}, \dot{x}=A(t) x\right\}, \\
\mathscr{X}_{l}:=\mathscr{X} \cap C_{l}, \quad \mathscr{X}_{l}^{+}:=\mathscr{X} \cap C_{l}^{+}, \quad \mathscr{X}_{l}^{-}:=\mathscr{X} \cap C_{l}^{-}, \\
\mathscr{X}_{l l}:=\mathscr{X} \cap C_{l l}, \quad \mathscr{X}_{0}:=\mathscr{X} \cap C_{0} .
\end{gathered}
$$

We treat the existence problem of the solutions for (3.2) in the following way. 
First, we search solutions in $C_{l}$ by using the well-known theory of admissibility. More exactly, we are looking for Banach spaces $B \subset C_{c}$ such that for every $b \in B$, (3.2) has solutions in $C_{l}$. Next, for these solutions belonging to $C_{l}$ we ask to satisfy the condition

$$
x(+\infty)=x(-\infty)
$$

and we try to find sufficient conditions such that the relation (3.4) becomes possible.

3.2. Consider now the scalar case

$$
\dot{x}=a(t) x+b(t),
$$

where $a, b: \mathbb{R} \rightarrow \mathbb{R}$ are continuous functions and $a, b \in C_{l}$.

Proposition 3.1. (1) If

$$
a(+\infty)<0, \quad a(-\infty)>0,
$$

then, for each $b \in C_{l}$ which satisfies

$$
a(+\infty) \cdot b(-\infty)-a(-\infty) \cdot b(+\infty)=0,
$$

every solution of (3.5) is homoclinic.

(2) If

$$
a(+\infty)>0, \quad a(-\infty)<0,
$$

then (3.5) admits one and only one solution in $C_{l}$ if and only if

$$
\int_{-\infty}^{+\infty} e^{-\int_{0}^{t} a(s) d s} \cdot b(t) d t=0 .
$$

If, furthermore, (3.7) is satisfied, this solution is homoclinic.

Proof. (1) We have

$$
x(t)=C \cdot e^{\int_{0}^{t} a(s) d s}+e^{\int_{0}^{t} a(s) d s} \cdot \int_{0}^{t} e^{-\int_{0}^{s} a(\tau) d \tau} \cdot b(s) d s .
$$

It results

$$
x( \pm \infty)=-\frac{b( \pm \infty)}{a( \pm \infty)} .
$$

(2) The only solution in $C_{l}^{+}$is

$$
x_{1}(t)=e^{\int_{0}^{t} a(s) d s} \cdot \int_{+\infty}^{t} e^{-\int_{0}^{s} a(\tau) d \tau} \cdot b(s) d s,
$$

and the unique solution in $C_{l}^{-}$is

$$
x_{2}(t)=e^{\int_{0}^{t} a(s) d s} \cdot \int_{-\infty}^{t} e^{-\int_{0}^{s} a(\tau) d \tau} \cdot b(s) d s .
$$


But, from (3.9) we obtain

$$
x_{1}(0)=x_{2}(0) \text {. }
$$

Hence, the solutions $x_{1}$ and $x_{2}$ are identical; the limits of these solutions to infinity are given by (3.11).

Corollary 3.2. (1) Assuming that (3.6) is true, for every $b \in C_{0}$, all the solutions of (3.5) are homoclinic to zero.

(2) Assuming that (3.8) is true, for every $b \in C_{0}$ which satisfies (3.9), (3.5) admits one and only one homoclinic solution.

Remark 3.3. Condition (3.9) can be interpreted as an orthogonality condition of $b$ on the solutions for the adjoint equation of the homogenous equation corresponding in (3.5).

3.3. The result contained in Proposition 3.1 can be generalized to the case of (3.2) in different ways; we exemplify here only one of them.

THEOREM 3.4. Suppose that

(i) $\lim _{t \rightarrow+\infty} A(t)=\left(\alpha_{i j}^{+}\right)_{i, j \in \overline{1, N}} ; \lim _{t \rightarrow-\infty} A(t)=\left(\alpha_{i j}^{-}\right)_{i, j \in \overline{1, N}}$;

(ii) $\alpha_{i i}^{+}<0, \alpha_{i i}^{-}>0$, for all $i \in \overline{1, N} ; \alpha_{i j}^{+}=\alpha_{i j}^{-}=0$, for all $i, j \in \overline{1, N}, i \neq j$.

Then,

(a) for each $b=\left(b_{i}\right)_{i \in \overline{1, N}} \in C_{l}$ which satisfies

$$
\alpha_{i i}^{+} \cdot b(-\infty)=\alpha_{i i}^{-} \cdot b(+\infty), \quad \forall i \in \overline{1, N},
$$

all the solutions of (3.2) are homoclinic,

(b) for each $b \in C_{0}$, all the solutions of (3.2) are homoclinic to zero.

We will sketch the proof, omitting the calculus details.

Let $A(t)=A_{1}(t)+\left[A(t)-A_{1}(t)\right]$, where

$$
A_{1}(t)=\operatorname{Diag}\left[a_{i i}(t)\right]_{i \in \overline{1, N}} .
$$

Consider $c \in \mathbb{R}^{N}$ fixed; for every $x \in C_{l}$, denote by $y=H x$ the unique solution for the problem

$$
\dot{y}=A_{1}(t) y+A_{2}(t) x(t)+b(t), \quad y(0)=c .
$$

By using Proposition 3.1 and hypotheses (i) and (ii), we get

$$
H C_{l} \subset C_{l} .
$$

Considering in $C_{l}$ a Bielecki norm

$$
\|H x\|_{\lambda}:=\sup _{t \in \mathbb{R}}\left\{|x(t)| \cdot e^{-\lambda|t|}\right\},
$$

for a positive real number $\lambda, H$ will result a contraction.

If $x=\left(x_{i}\right)_{i \in \overline{1, N}} \in C_{l}$ is the unique fixed point of $H$, then we can apply Proposition 3.1 to every $x_{i}, i \in \overline{1, N}$. 
3.4. Another possibility for obtaining existence results in $C_{l}$ is based on the remark that if $x$ is of class $C^{1}$, then $x$ belongs to $C_{l}$ if and only if $\dot{x}$ is Riemann integrable on $\mathbb{R}$.

We also use in this subsection the notations

$$
\mathscr{X}^{*}:=\left\{x, \dot{x}(t)=A^{*}(t) x(t)\right\}, \quad \mathscr{X}_{l}^{*}=\mathscr{X}^{*} \cap C_{l}, \quad \mathscr{X}_{l l}^{*}=\mathscr{X}^{*} \cap C_{l l} .
$$

Proposition 3.5. Suppose that

$$
\int_{-\infty}^{+\infty}|A(t)| d t<+\infty .
$$

Then,

(a) $\mathscr{L}=\mathscr{X}_{l}$ and $\mathscr{L}^{*}=\mathscr{X}_{l}^{*}$;

(b) $X( \pm \infty)$ are invertible;

(c) $\operatorname{dim} \mathscr{X}_{l l}=\operatorname{dim} \mathscr{X}_{l l}^{*}$.

Proof. Let $x \in \mathscr{X}$; from the classical inequality

$$
|x(t)| \leq|x(0)| \cdot e^{\left|\int_{0}^{t}\right| A(s)|d s|}, \quad \forall t \in \mathbb{R},
$$

we obtain $x \in C$; but since

$$
\dot{x}(t)=A(t) \cdot x(t)
$$

and (3.21) is true, it follows that $\dot{x}$ is Riemann integrable on the whole real axis $\mathbb{R}$. Therefore, (a) is obvious.

Denote $\Delta(t):=\operatorname{det} X(t)$. Since the well-known identity

$$
\Delta(t)=\Delta(0) \cdot e^{\int_{0}^{t} \operatorname{Trace}(A(s)) d s}
$$

and (3.21) hold, we conclude that

$$
\operatorname{det} X( \pm \infty):=\lim _{t \rightarrow \pm \infty} \Delta(t) \neq 0 .
$$

Hence, it is easy to observe that

$$
\mathscr{X}_{l l}=\{X(t) \cdot c,[X(+\infty)-X(-\infty)] \cdot c=0\},
$$

so the conclusion (c) is immediate, too.

Remark 3.6. We have $\mathscr{X}_{l l}=\{0\}$ if and only if

$$
\operatorname{rank}[X(+\infty)-X(-\infty)]=N
$$

which is equivalent to the fact that 1 is not an eigenvalue of the matrix $X^{-1}(-\infty)$. $X(+\infty)$.

THEOREM 3.7. If condition (3.21) is fulfilled, then for each $b \in L^{1} \cap C_{c}$, all the solutions of (3.2) belong to $C_{l}$. 
Furthermore, (3.2) has homoclinic solutions if and only if

$$
\int_{-\infty}^{+\infty}\langle b(s) \mid y(s)\rangle d s=0
$$

where $y$ is any solution of the problem

$$
\dot{y}=A^{*} y, \quad y(-\infty)=y(+\infty) .
$$

Proof. By using Proposition 3.5 and the identity

$$
X(t) \cdot X^{-1}(t)=I,
$$

it follows that $X^{-1}(t)$ is bounded.

Let $x$ be a solution of (3.2); then

$$
x(t)=X(t) \cdot x(0)+X(t) \cdot \int_{0}^{t} X^{-1}(s) \cdot b(s) d s .
$$

We get $x \in C$ and from

$$
\dot{x}(t)=A(t) \cdot x(t)+b(t),
$$

it results that $\dot{x} \in L^{1}, x \in C_{l}$.

Of course, (3.31) is a homoclinic solution with $x(0)=c$ which implies

$$
\begin{aligned}
{[X(+\infty)-X(-\infty)] \cdot c=} & X(+\infty) \cdot \int_{0}^{+\infty} X^{-1}(s) \cdot b(s) d s \\
& +X(-\infty) \cdot \int_{-\infty}^{0} X^{-1}(s) \cdot b(s) d s
\end{aligned}
$$

But, the relation (3.33) is exactly the sufficient and necessary compatibility condition for this system.

3.5. Another possibility to obtain the existence of homoclinic solutions would be the reduction of our problem to an integral equation by using a Green's function, the treatment being like in the theory of bilocal boundary value problems.

The admissibility with respect to an integral operator has been considered first by C. Corduneanu [10] and D. Petrovanu [21]. The admissibility hypothesis on the pair $\left(C_{l}, C_{l}\right)$ is developed in [4].

The building of the Green's function which will be used is based on the decomposition of $\mathbb{R}^{N}$ into a direct sum

$$
\mathbb{R}^{N}=\mathbb{R}_{0} \oplus \mathbb{R}_{-} \oplus \mathbb{R}_{+} \oplus \mathbb{R}_{S}
$$

where

$$
\mathbb{R}_{0}:=\left\{x(0), x \in \mathscr{X}_{l}\right\}, \quad \mathbb{R}_{-}:=\left\{x(0), x \in \mathscr{X}_{l}^{+}\right\}, \quad \mathbb{R}_{+}:=\left\{x(0), x \in \mathscr{X}_{l}^{-}\right\} .
$$

So, $\mathbb{R}_{S}$ is the supplement of $\mathbb{R}_{0} \oplus \mathbb{R}_{-} \oplus \mathbb{R}_{+}$. 
74 Homoclinic solutions

Denote by $P_{0}, P_{-}, P_{+}, P_{s}$ some projectors in $\mathbb{R}^{N}$ which are associated to these subspaces $\mathbb{R}_{0}, \mathbb{R}_{-}, \mathbb{R}_{+}, \mathbb{R}_{s}$.

Define the function $\mathscr{G}: \mathbb{R}^{2} \rightarrow \mu_{N}(\mathbb{R})$ by

$$
\mathscr{G}(t, s):= \begin{cases}X(t) P_{-} X^{-1}(s), & \text { for } s \leq t \leq 0, \\ -X(t)\left(P_{0}+P_{+}\right) X^{-1}(s), & \text { for } t \leq s \leq 0, \\ -X(t) P_{+} X^{-1}(s), & \text { for } t \leq 0 \leq s, \\ X(t) P_{-} X^{-1}(s), & \text { for } s \leq 0 \leq t, \\ X(t)\left(P_{0}+P_{-}\right) X^{-1}(s), & \text { for } 0 \leq s \leq t, \\ -X(t) P_{+} X^{-1}(s), & \text { for } 0 \leq t \leq s .\end{cases}
$$

The function $\mathscr{G}=\mathscr{G}(t, s)$ is continuous in the set

$$
\mathbb{R}^{2} \backslash\{(t, t), t \in \mathbb{R}\}
$$

Consider the integral operator

$$
G b:=\int_{-\infty}^{+\infty} \mathscr{G}(t, s) \cdot b(s) d s .
$$

The fundamental properties of this operator are given by the following proposition.

Proposition 3.8. If

$$
\int_{-\infty}^{+\infty}|\varphi(t, s)| d s \leq k, \quad \forall t \in \mathbb{R}
$$

then

$$
G C_{0} \subset C_{0}
$$

If, furthermore,

$$
\exists \lim _{t \rightarrow \pm \infty} \int_{-\infty}^{+\infty} \mathscr{G}(t, s) d s=L_{ \pm} \in \mathcal{M}_{N}(\mathbb{R}),
$$

then

$$
\begin{gathered}
G C_{l} \subset C_{l}, \\
\lim _{t \rightarrow \pm \infty} \int_{-\infty}^{+\infty} \mathscr{G}(t, s) \cdot b(s) d s=L_{ \pm} \cdot b( \pm \infty) .
\end{gathered}
$$

Moreover, the operator $G: C_{l} \rightarrow C_{l}$ is compact.

Proof. We study the asymptotic behavior of the function $(G b)(t)$ at $+\infty$; at $-\infty$ the proof is identical. 
First, remark that from (3.39) it results for $t \geq 0$ the following inequalities:

$$
\begin{aligned}
\int_{-\infty}^{0}\left|X(t) P_{-} X^{-1}(s)\right| d s \leq k, & \forall t \geq 0, \\
\int_{0}^{t}\left|X(t)\left(P_{0}+P_{-}\right) X^{-1}(s)\right| d s \leq k, & \forall t \geq 0, \\
\int_{t}^{+\infty}\left|X(t) P_{+} X^{-1}(s)\right| d s \leq k, & \forall t \geq 0 .
\end{aligned}
$$

Applying Proposition 2.5 with $U(t)=X(t)$, we get since (3.45),

$$
\lim _{t \rightarrow+\infty}\left|X(t)\left(P_{0}+P_{-}\right)\right|=0
$$

and since (3.44), (3.46) for $t=0$,

$$
\int_{-\infty}^{0}\left|P_{-} X^{-1}(s)\right| d s \leq k, \quad \int_{0}^{+\infty}\left|P_{+} X^{-1}(s)\right| d s \leq k .
$$

But, for $t \geq 0$, we have

$$
\begin{aligned}
(G b)(t)= & \int_{-\infty}^{0} X(t) P_{-} X^{-1}(s) b(s) d s+\int_{0}^{t} X(t)\left(P_{0}+P_{-}\right) X^{-1}(s) b(s) d s \\
& -\int_{t}^{+\infty} X(t) P_{+} X^{-1}(s) b(s) d s .
\end{aligned}
$$

Let $b \in C_{0}$; since

$$
\begin{aligned}
& \left|\int_{-\infty}^{0} X(t) P_{-} X^{-1}(s) b(s) d s\right| \leq \int_{-\infty}^{0}\left|X(t) P_{-}\right| \cdot\left|P_{-} X^{-1}(s)\right| \cdot|b(s)| d s \\
& \leq\left|X(t) P_{-}\right| \cdot k \cdot\|b\|_{\infty}, \\
& \left|\int_{t}^{+\infty} X(t) P_{+} X^{-1}(s) b(s) d s\right| \leq k \cdot \sup _{s \geq t}|b(s)|, \\
& \left|\int_{0}^{t} X(t)\left(P_{0}+P_{-}\right) X^{-1}(s) b(s) d s\right| \leq\left|X(t)\left(P_{0}+P_{-}\right)\right| \int_{0}^{T}\|x\|_{\infty}\left|X^{-1}(s)\right| d s \\
& +k \cdot \sup _{t \geq T}|b(t)|, \quad \text { for } 0<T<t,
\end{aligned}
$$

we obtain

$$
\lim _{t \rightarrow+\infty}(G b)(t)=0 .
$$

The conclusion (3.43) is proved because every $b \in C_{l}$ can be written under the form

$$
b(t)=[b(t)-b(+\infty)]+b(+\infty) .
$$

Finally, the compactness of $G$ is obtained from Proposition 2.4. 
Remark 3.9. The conditions (3.39) and (3.41) are also neccesary for the embedding $G C_{l} \subset C_{l}$.

Remark 3.10. In general, $G b$ is not a solution for (3.2). If $P_{s} b(t)=0$, for all $t \in \mathbb{R}$, then $(G b)(t)$ is a solution of (3.2). Moreover, it is the only solution which satisfies

$$
P_{0} x(0)=0 .
$$

Remark 3.11. The relation (3.39) allows us (by using Proposition 2.6) to elucidate the asymptotic behavior of the solutions for the homogenous equation (3.1), in the case when $P_{s}=0$ :

(a) $\mathscr{X}_{l}=\mathscr{L}_{0}=\left\{x \in \mathscr{X}, x(0) \in \mathbb{R}_{0}\right\}$;

(b) $x \in \mathscr{X}$ and $x(0) \in \mathbb{R}_{-}$imply limsup $\sup _{t \rightarrow-\infty}|x(t)|=+\infty$;

(c) $x \in \mathscr{L}$ and $x(0) \in \mathbb{R}_{+}$imply $\lim \sup _{t \rightarrow+\infty}|x(t)|=+\infty$.

By using Remark 3.10, we consider the following spaces:

$$
\begin{gathered}
B_{0}:=\left\{b \in C_{0}, P_{s} b(t)=0, \forall t \in \mathbb{R}\right\}, \\
B_{l}:=\left\{b \in C_{l}, P_{s} b(t)=0, \forall t \in \mathbb{R}\right\}, \\
B_{l l}:=B_{l} \cap C_{l l} .
\end{gathered}
$$

Now we attach to (3.2) the "initial" condition

$$
P_{0} x(0)=\xi, \quad \xi \in \mathbb{R}_{0} .
$$

Theorem 3.12. Suppose that conditions (3.39) and (3.41) are fulfilled. Then:

(a) for every $b \in B_{0}$ and $\xi \in \mathbb{R}_{0}$, all the solutions of the problem (3.2) and (3.55) are homoclinic to zero;

(b) for every $b \in B_{l}$ which satisfies

$$
L_{+} b(+\infty)=L_{-} b(-\infty)
$$

the solutions of the problem (3.2) and (3.55) are homoclinic;

(c) if $L_{+}=L_{-}$, then for every $b \in B_{l l}$, the solutions of the problem (3.2) and (3.55) are homoclinic;

(d) if $L_{+}=L_{-}=0$, then for every $b \in B_{l}$, the solutions of the problem (3.2) and (3.55) are homoclinic.

The conclusions of this theorem are immediate since Proposition 3.8 holds and for $b \in B_{0}$ the solution of (3.2) and (3.55) is given by

$$
x(t)=X(t) \xi+(G b)(t), \quad \xi \in \mathbb{R}_{0} .
$$

3.6. A short version of Theorem 3.12 is the following, where the conditions are given on the positive semiaxis.

Theorem 3.13. Suppose that

(i) $A(-t)=-A(t)$, for all $t \geq 0$; 
(ii) $\int_{0}^{t}\left|X(t) \cdot X^{-1}(s)\right| d s \leq k$, for all $t \geq 0$;

(iii) $\exists L:=\lim _{t \rightarrow+\infty} \int_{0}^{t} X(t) \cdot X^{-1}(s) d s$.

Then, for every $b \in C_{l l}$, all the solutions of (3.2) are homoclinic.

Proof. The conditions (i) and (ii) allow us to conclude that every solution $x$ of (3.2) belongs to $C_{l}^{+}$and

$$
x(+\infty)=L \cdot b(+\infty)
$$

But, the function $y(t):=x(-t)$ is a solution of

$$
\dot{y}=A(t) \cdot y+b(-t) \text {. }
$$

So,

$$
y(+\infty)=L \cdot b(-\infty)
$$

But,

$$
y(+\infty)=x(-\infty)
$$

The proof is now complete.

3.7. The proof of Proposition 3.8 contains separately the asymptotic behavior research of the solutions at $+\infty$ and next at $-\infty$.

The following proposition points out another aspect in this direction.

Proposition 3.14. Let $B \subset C_{c}$ be an arbitrary Banach space. Suppose that

(i) for every $b \in B$, (3.2) has solutions in $C_{l}^{+}$;

(ii) for every $b \in B$, (3.2) has solutions in $C_{l}^{-}$;

(iii) $\mathbb{R}_{s}=\{0\}$.

Then, (3.2) admits homoclinic solutions.

Proof. From (iii) it results

$$
\mathscr{X}=X_{l}^{+} \oplus \mathscr{X}_{l}^{-} \oplus \mathscr{X}_{l l} .
$$

Let $b \in B$ and let $y_{+} \in C_{l}^{+}, y_{-} \in C_{l}^{-}$be the solutions given by (i) and (ii). From $y_{+}-y_{-} \in \mathscr{X}$ it results

$$
y_{+}-x_{+}=y_{-}+x_{-}+x_{0}
$$

where

$$
x_{+} \in \mathscr{X}_{l}^{+}, \quad x_{-} \in \mathscr{X}_{l}^{-}, \quad x_{0} \in \mathscr{X}_{l l} .
$$

Let $z:=y_{+}-x_{+}, \tilde{z}:=y_{-}+x_{-}+x_{0}$. Because $z \in C_{l}^{+}, \tilde{z} \in C_{l}^{-}$, and $z(0)=\tilde{z}(0)$, one gets

$$
z=\tilde{z}
$$

Using the previous proposition we obtain the following theorem. 
Theorem 3.15. Suppose that

(i) for every $b \in B$, (3.2) has solutions in $C_{0}^{+}$;

(ii) for every $b \in B$, (3.2) has solutions in $C_{0}^{-}$;

(iii) $\mathscr{X}=\mathscr{X}_{l}^{+} \oplus \mathscr{X}_{l}^{-} \oplus \mathscr{X}_{l l}$;

(iv) for every $x \in \mathscr{X}, x=x_{+}+x_{-}+x_{l l}$ it follows

$$
x_{+}(+\infty)+x_{-}(-\infty)+x_{l l}(+\infty)=0 .
$$

Then, (3.2) admits homoclinic solutions.

3.8. We point out the fact that $C_{l}$ and $C_{(a, b)}$ are isomorphic, like in Proposition 2.1; for simplification and to achieve a similarity with a classical problem, we take $a=0$ and $b=\omega$.

Let $\varphi: \mathbb{R} \rightarrow(0, \omega)$ be a $C^{1}$ function satisfying the conditions

$$
\dot{\varphi}(t)>0, \quad \forall t \in \mathbb{R}, \quad \varphi(-\infty)=0, \quad \varphi(+\infty)=\omega .
$$

We put for simplification, $\psi^{-1}=\varphi$.

Consider first the homogenous equation

$$
\dot{x}=A(t) x .
$$

Proposition 3.16. Suppose that

$$
\lim _{t \rightarrow \pm \infty} \frac{1}{\dot{\varphi}(t)} \cdot A(t)=\Lambda_{ \pm} \in \mathcal{M}_{N}(\mathbb{R}) .
$$

Then,

$$
\mathscr{X}_{l}=\mathscr{X}
$$

Proof. We put

$$
C(t)= \begin{cases}\dot{\psi}(t) \cdot A[\psi(t)], & \text { for } t \in(0, \omega), \\ \Lambda_{+}, & \text {for } t=\omega, \\ \Lambda_{-}, & \text {for } t=0 .\end{cases}
$$

Obviously, $C(t)$ is continuous on $[0, \omega]$; let $y(s)$ be a solution of

$$
\dot{y}=C(s) \cdot y, \quad \forall s \in[0, \omega] .
$$

Then, $x(t):=y(\varphi(t))$ is a solution for (3.1); moreover, $x(+\infty)=y(\omega), x(-\infty)=$ $y(0)$.

The following result is now obvious.

THEOREM 3.17. If the boundary value problem

$$
\dot{y}=C(s) y+d(s), \quad y(0)=y(\omega),
$$

has a solution for $d \in C_{(a, b)}$ and if the relation (3.69) is satisfied, then (3.2) admits homoclinic solutions for $b(t)=(1 / \dot{\varphi}(t)) \cdot d(\varphi(t))$. 
For the problem (3.73) do exist many existence results; by using the mentioned way, these results can be used to prove the existence of the homoclinic solutions for (3.2).

We end this section with an interesting result deduced from (3.69).

Proposition 3.18. Suppose that (3.69) is true, with $\Lambda_{+}=\Lambda_{-}$. Let $D \in M_{N}(\mathbb{R})$ such that

$$
\tilde{X}(+\infty)=e^{D \omega},
$$

where $\tilde{X}$ is the fundamental matrix of (3.1) with $\tilde{X}(-\infty)=I$. Then the transformation

$$
x(\psi(t))=\tilde{X}(\psi(t)) e^{-D t} z(t)
$$

transforms our problem into the linear equation with constant coefficients

$$
\dot{z}=D z
$$

Proof. The transformation $x(t) \mapsto y(t):=x(\psi(t))$ transforms (3.1) into (3.72); since $C(0)=C(\omega)$, the matrix $C(t)$ can be prolonged by periodicity to the whole real axis $\mathbb{R}$.

Let $Y(t)$ be the fundamental matrix of (3.72) with $Y(0)=I$; then

$$
Y(s)=\tilde{X}(\psi(s)),
$$

and (3.74) becomes

$$
Y(\omega)=e^{D \omega} .
$$

But a classical result enables us to conclude that the transformation

$$
y(t)=Y(t) \cdot e^{-D t} \cdot z(t)
$$

transforms (3.72) into (3.76).

\section{Linearizable equations}

4.1. In this section, we consider the nonlinear problem

$$
\begin{gathered}
\dot{x}=F(t, x), \\
x(-\infty)=x(+\infty),
\end{gathered}
$$

for certain categories of functions $F$ and the corresponding equations will be called linearizable.

We treat only two linearizable cases which will be named differential linearizability and homotopic linearizability.

4.2. Let $A: \mathbb{R} \times \mathbb{R}^{N} \rightarrow \mu_{N}(\mathbb{R})$ and $f: \mathbb{R} \times \mathbb{R}^{N} \rightarrow \mathbb{R}^{N}$ be two continuous functions; consider the case

$$
F(t, x)=A(t, x) x+f(t, x)
$$


in this case we say that (4.1) is differential linearizable; it is clear that every differentiable function $F$ can be written under the form (4.3).

We precise the notations and the general hypotheses.

Denote $\Sigma_{r}:=\left\{x \in \mathbb{R}^{N},|x| \leq r\right\}, S_{r}:=\left\{x \in C_{0},\|x\|_{\infty} \leq r\right\}$.

Let $P: \mathbb{R}^{N} \rightarrow \mathbb{R}^{N}$ be an arbitrary projector, $A: \mathbb{R} \times \mathbb{R}^{N} \rightarrow \mu_{N}(\mathbb{R})$ and $f: \mathbb{R} \times$ $\mathbb{R}^{N} \rightarrow \mathbb{R}^{N}$ be continuous functions.

We admit the following hypotheses:

(A1) $A$ is uniformly continuous on $\mathbb{R} \times \Sigma_{r}$;

(A2) $A(t, x)=-A(-t, x), \forall t \in \mathbb{R}, \forall x \in \Sigma_{r}$;

(f1) $\forall u \in S_{r}$, the function $f(\cdot, u(\cdot)) \in C_{0}$;

(f2) $(I-P) f(t, u(t))=0, \forall u \in S_{r}, \forall t \in \mathbb{R}$;

(f3) $\limsup _{x \rightarrow 0}(|f(t, x)| /|x|)=0$, uniformly with respect to $t \in \mathbb{R}$.

Theorem 4.1. Suppose that the fundamental matrix $X_{0}(t)$ of the system

$$
\dot{x}=A(t, 0) x
$$

admits an exponential dichotomy on $\mathbb{R}_{+}$by the form

$$
\begin{aligned}
\left|X_{0}(t) P X_{0}^{-1}(s)\right| \leq k \cdot e^{-\alpha(t-s)}, & \text { for } 0 \leq s \leq t, \\
\left|X_{0}(t)(I-P) X_{0}^{-1}(s)\right| \leq k \cdot e^{-\alpha(s-t)}, & \text { for } 0 \leq t \leq s,
\end{aligned}
$$

where $\alpha>0, k \geq 1$.

Then, the problem

$$
\dot{x}=A(t, x) x+f(t, x), \quad P x(0)=\xi, \quad \xi \in P \mathbb{R}^{N},
$$

has homoclinic solutions to zero, if $|\xi|$ is small enough.

Proof. The hypothesis (A2) ensures us a similar behavior on $\mathbb{R}_{-}$for $X_{0}(t)$.

From (A1) it results that there exists $r_{0}<r$ such that

$$
|A(t, x)-A(t, 0)|<\frac{\alpha}{36 k^{5}}, \quad \forall x \in \Sigma_{r_{0}}, \forall t \in \mathbb{R} .
$$

Denote

$$
\delta:=\sup \left\{|A(t, x)-A(t, 0)|, x \in \Sigma_{r_{0}}, t \in \mathbb{R}\right\} .
$$

But then (see [9]) for every $u \in \Sigma_{r_{0}}$ the equation

$$
\dot{x}=A(t, u(t)) x
$$

admits a fundamental matrix $X_{u}(t)$ such that

$$
\begin{aligned}
\left|X_{u}(t) P X_{u}^{-1}(s)\right| \leq k_{1} \cdot e^{-\alpha_{1}(t-s)}, & \text { for } 0 \leq s \leq t, \\
\left|X_{u}(t)(I-P) X_{u}^{-1}(s)\right| \leq k_{1} \cdot e^{-\alpha_{1}(s-t)}, & \text { for } 0 \leq t \leq s,
\end{aligned}
$$

where $k_{1}=12 k^{3}, \alpha_{1}=\alpha-6 k^{3} \delta$. 

form

The hypothesis (A2) enables us to extend the previous inequalities to $\mathbb{R}$ under the

$$
\begin{aligned}
\left|X_{u}(t) P X_{u}^{-1}(s)\right| \leq k_{1} \cdot e^{-\alpha_{1}|t-s|}, & & \text { for }|s| \leq|t|, \\
\left|X_{u}(t)(I-P) X_{u}^{-1}(s)\right| \leq k_{1} \cdot e^{-\alpha_{1}|t-s|}, & & \text { for }|t| \leq|s| .
\end{aligned}
$$

Let $\varepsilon<\alpha_{1} / k_{1}$; by using the hypothesis (f3) there exists $\rho<r_{0}$ such that

$$
|f(t, x)| \leq \varepsilon \rho, \quad \forall x \in \Sigma_{r_{0}}, \forall t \in \mathbb{R} .
$$

Define on $S_{\rho} \subset C_{0}$ the operator

$$
(H u)(t):=X_{u}(t) P \xi+\int_{0}^{t} X_{u}(t) P X_{u}^{-1}(s) f(s, u(s)) d s .
$$

Using Theorem 3.12 with $P_{-}=P_{+}=0, P_{0}=P$, we get

$$
H S_{\rho} \subset C_{0}
$$

furthermore, $H$ is compact.

A simple calculation shows us that

$$
|\xi|<\rho \cdot\left(\frac{1}{k_{1}}-\frac{1}{\alpha_{1}} \cdot \varepsilon\right)
$$

implies the embedding $H S_{\rho} \subset S_{\rho}$.

All the hypotheses of Schauder's fixed point theorem being satisfied, $H$ has at least a fixed point. The proof is now complete.

4.3. We return now to the problem

$$
\begin{gathered}
\dot{x}=F(t, x), \\
x(-\infty)=x(+\infty) .
\end{gathered}
$$

In [2] were introduced the so-called associated operators to the problem (4.16) and (4.17): an operator $K$, defined on a subset of $C_{l}$ (or $C_{l l}$ ) is called associated if it is compact and if its fixed points coincide with the solutions of the problem (4.16) and (4.17). The easiest example for such an associated operator is

$$
(K x)(t)=x(+\infty)+\int_{-\infty}^{t} F(s, x(s)) d s .
$$

The associated operators exist always if the following conditions are fulfilled:

(F1) $F: \mathbb{R} \times \mathbb{R}^{N} \rightarrow \mathbb{R}^{N}$ is continuous;

(F2) the set $\left\{F(t, x(t)), x \in B_{\rho}\right\}$ is bounded in $L^{1}$, where

$$
B_{\rho}:=\left\{x \in C_{l},\|x\|_{\infty} \leq \rho\right\} .
$$


Definition 4.2. We say that the function $F$ is homotopic linearizable on $B_{\rho}$ if there exists a continuous function $A: \mathbb{R} \rightarrow M_{N}(\mathbb{R})$ such that if for a $\lambda \in[0,1]$, the problem

$$
\dot{x}=\lambda A(t) x+(1-\lambda) F(t, x), \quad x(-\infty)=x(+\infty),
$$

has a solution $x$, then $\|x\|_{\infty}<\rho$.

Theorem 4.3. Assuming that

(i) (F1) and (F2) are satisfied;

(ii) $F$ is homotopic linearizable on $B_{\rho}$;

(iii) $\int_{-\infty}^{+\infty}|A(t)| d t<+\infty$;

(iv) $\operatorname{det}[X(+\infty)-X(-\infty)] \neq 0$,

then (4.16) admits homoclinic solutions.

Proof. Let $K_{\lambda}=K_{\lambda}(x)$ be an associated operator for the problem (4.20) on $B_{\rho}$. From hypotheses (i) and (ii) and the homotopic invariance property of the topological degree, it follows that

$$
\operatorname{deg}\left(I-K_{0}, B_{\rho}, 0\right)=\operatorname{deg}\left(I-K_{1}, B_{\rho}, 0\right) .
$$

Using Theorem 3.7 and hypothesis (iv) we easily obtain that the operator $K_{1}$ is compact and $I-K_{1}$ is injective; since $0 \in B_{\rho}$, a classical result in topological degree theory leads us to the equality

$$
\operatorname{deg}\left(I-K_{1}, B_{\rho}, 0\right)= \pm 1
$$

Therefore,

$$
\operatorname{deg}\left(I-K_{0}, B_{\rho}, 0\right) \neq 0,
$$

and so $K_{0}$ admits a fixed point in $B_{\rho}$. Since $K_{0}$ is the associated operator of the problems (4.16), (4.17), the theorem is proved.

\section{References}

[1] C. Avramescu, Existence problems for w-closed orbits, to appear in Commun. Appl. Anal.

[2] _ Topological degree and homoclinic solutions, to appear in Ann. Mat. Pura Appl.

[3] _ Sur l'existence des solutions convergentes des systèmes d'équations différentielles non linéaires, Ann. Mat. Pura Appl. (4) 81 (1969), 147-168. MR 40\#2979. Zbl 196.10701.

[4] __ Sur l'existence des solutions des équations intégrales dans certains espaces fonctionnels, Ann. Univ. Sci. Budapest. Eötvös Sect. Math. 13 (1970), 19-34 (1971) (French). MR $47 \# 737$.

[5] __ Sur l'admissibilité par rapport à un opérateur intégral linéaire, An. Şti. Univ. "Al. I. Cuza" Iaşi Secpolhk t. I a Mat. (N.S.) 18 (1972), 55-64. MR 48\#2690. Zbl 367.47027.

[6] Sur l'existence des solutions convergentes pour des équations intégrales, An. Univ. Craiova Ser. a V-a (1974), no. 2, 87-98 (French). MR 54\#8184. Zbl 306.45015.

[7]_, Quelques remarques concernant l'existence et l'approximation des solutions d'une équation non linéaire dans un espace de Banach [Some remarks concerning the existence and approximation of solutions of a nonlinear equation in Banach space], An. Univ. Craiova Ser. Mat. Inform. 22 (1995), 14-23 (1997) (French). MR 99k:47155. 
[8] __ Sur le degré topologique attaché à une équation différentielle définie dans l'espace des fonctions convergentes [On the topological degree associated with a differential equation defined in the space of convergent functions], An. Univ. Timişoara Ser. Mat.-Inform. 34 (1996), no. 2, 181-187 (French). MR 99i:34082.

[9] M. Cecchi, M. Marini, and P. L. Zezza, An abstract method for nonlinear boundary value problems on noncompact intervals, Ordinary Differential Equations and Functional Equations, CNR, Rome, 1978, (Florence, 1978), pp. 395-405. MR 84f:34016.

[10] C. Corduneanu, Problèmes globaux dans la théorie des équations intégrales de Volterra, Ann. Mat. Pura Appl. (4) 67 (1965), 349-363. MR 32\#331. Zbl 151.16801.

[11] T. G. Hallam, Convergence of solutions of perturbed nonlinear differential equations, Ann. Mat. Pura Appl. (4) 94 (1972), 275-282. MR 47\#3782. Zbl 283.34052.

[12] On nonlinear functional perturbation problems for ordinary differential equations, J. Differential Equations 12 (1972), 63-80. MR 48\#6574. Zbl 243.34015.

[13] A. G. Kartsatos, Convergence in perturbed nonlinear systems, Tôhoku Math. J. (2) 24 (1972), 539-546. MR 48\#2508. Zbl 245.34028.

[14] _ Existence of solutions of heavily nonlinear Volterra integral equations, Hiroshima Math. J. 3 (1973), 243-249. MR 49\#11168. Zbl 303.45004.

[15] _ Positive solutions to nonlinear systems, J. Differential Equations (1974), 330-341.

[16] _ Advanced Ordinary Differential Equations, 2nd ed., Mancorp Publishing, Florida, 1993. MR 94m:34001. Zbl 816.34001.

[17] J. Mawhin, Topological Degree Methods in Nonlinear Boundary Value Problems, CBMS Regional Conference Series in Mathematics, vol. 40, American Mathematical Society, Rhode Island, 1979. MR 80c:47055. Zbl 414.34025.

[18] _ Topological degree and boundary value problems for nonlinear differential equations, Topological Methods for Ordinary Differential Equations, (Montecatini Terme, 1991), vol. 1537, Springer, Berlin, 1993, Lecture Notes in Math., pp. 74-142. MR Zbl ..798.34025

[19] _ Continuation theorems and periodic solutions of ordinary differential equations, Topological Methods in Differential Equations and Inclusions, NATO Adv. Sci. Inst. Ser. C Math. Phys. Sci., vol. 472, Kluwer Acad. Publ., Dordrecht, 1995, (Montreal, PQ, 1994), pp. 291-375. MR 96m:34074. Zbl 834.34047.

[20] Leray-Schauder continuation theorems in the absence of a priori bounds, Topol. Methods Nonlinear Anal. 9 (1997), no. 1, 179-200. MR 99m:47075. Zbl 912.47040.

[21] D. Petrovanu, Équations Hammerstein intégrales et discrètes, Ann. Mat. Pura Appl. (4) 74 (1966), 227-254 (French). MR 35\#687. Zbl 144.15901.

C. Avramescu: Department of Mathematics, University of Craiova, A.I. Cuza Street

13, Craiova 1100, Romania

E-mail address: cezaravramescu@hotmail.com

C. Vladimirescu: Department of Mathematics, University of Craiova, A.I. Cuza Street

13, Craiova 1100, Romania

E-mail address: vladimirescucris@ hotmail.com 


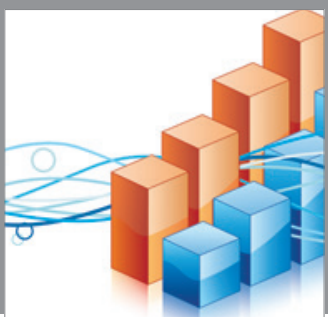

Advances in

Operations Research

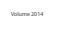

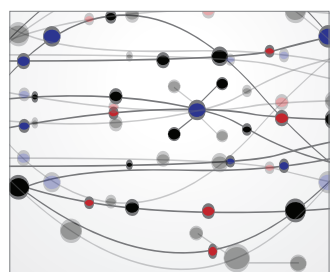

\section{The Scientific} World Journal
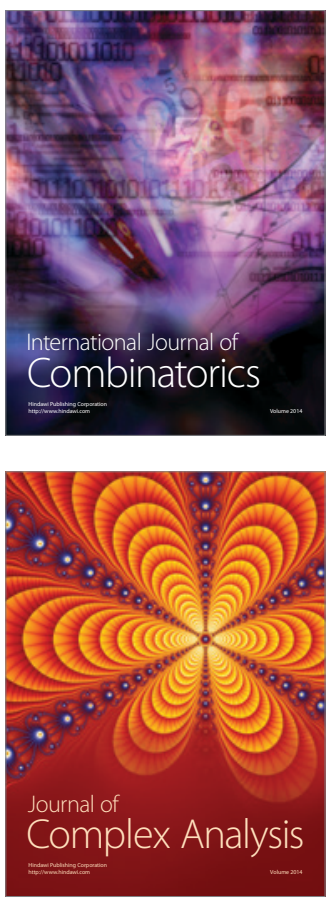

International Journal of

Mathematics and

Mathematical

Sciences
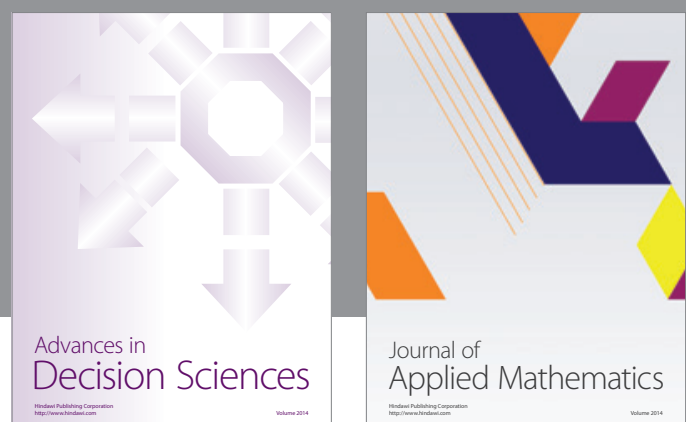

Journal of

Applied Mathematics
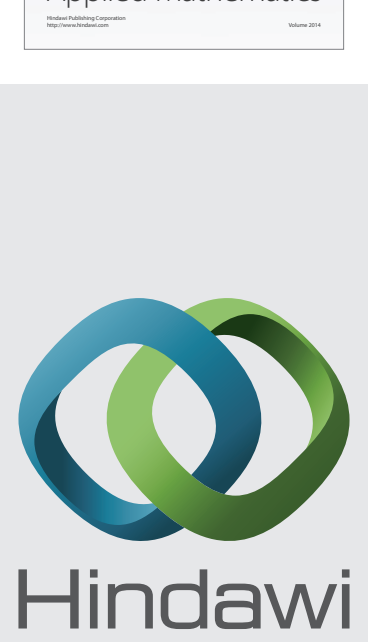

Submit your manuscripts at http://www.hindawi.com
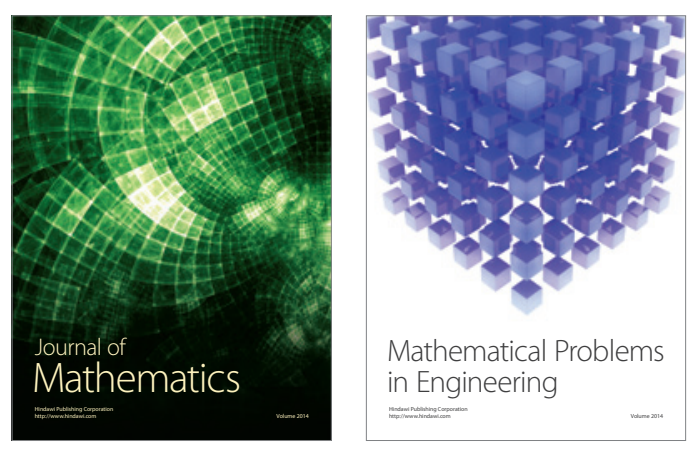

Mathematical Problems in Engineering
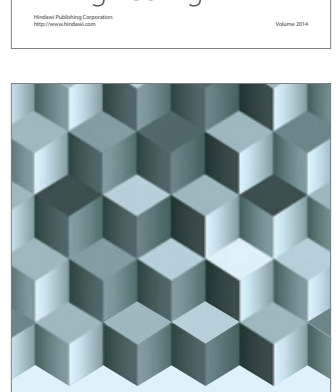

Journal of

Function Spaces
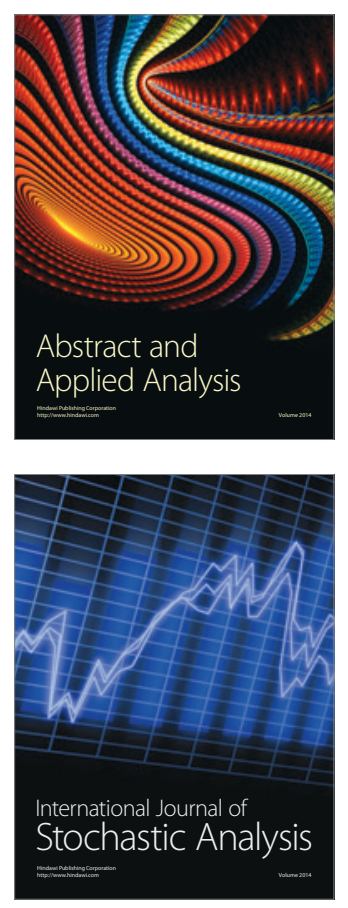

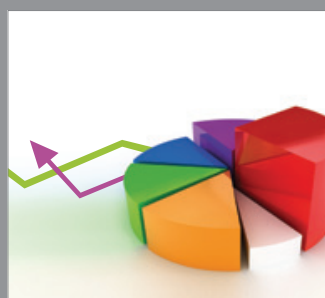

ournal of

Probability and Statistics

Promensencen
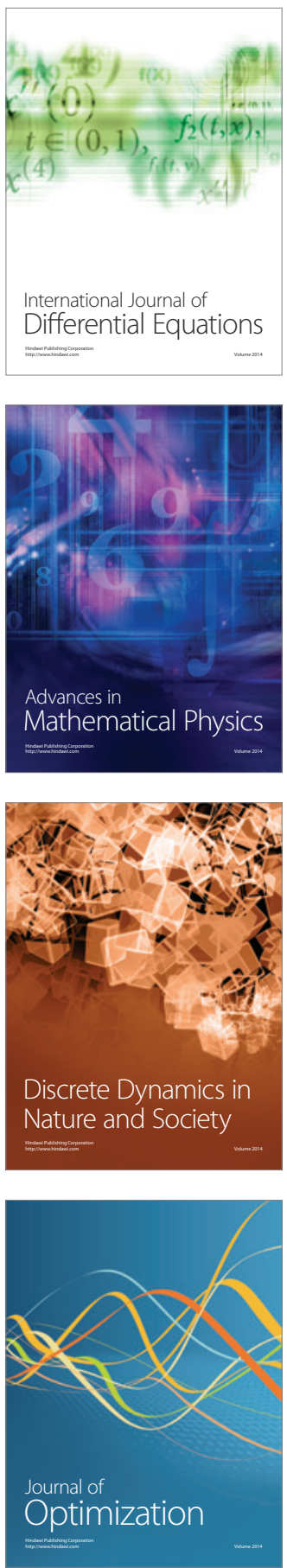